\title{
SIMULAÇÃO DE GASEIFICAÇÃO DE BIOMASSA DE TAMAREIRA PARA PRODUÇÃO DE GÁS DE SÍNTESE
}

\author{
A. E. de OLIVEIRA ${ }^{1}$, J. B. O. SANTOS ${ }^{1}$ \\ ${ }^{1}$ Universidade Federal de São Carlos, Departamento de Engenharia Química \\ E-mail para contato: joaobatista@ufscar.br
}

\begin{abstract}
RESUMO - A simulação do processo de gaseificação da biomassa de tamareira foi realizada utilizando-se o software Aspen Plus 8.4 com o objetivo de verificar a influência da temperatura do gaseificador e da razão vapor-biomassa na composição de saída dos produtos $\mathrm{H}_{2}, \mathrm{CO}, \mathrm{CO}_{2}$ e $\mathrm{CH}_{4}$. Foi verificado que a temperatura tem pouca influência sobre a produção de $\mathrm{H}_{2}$, mas afeta a formação de $\mathrm{CO}$ e $\mathrm{CO}_{2}$. A razão vapor-biomassa tem pouca influência na formação de $\mathrm{H}_{2}$, mas o aumento dessa razão afeta a produção de $\mathrm{CO}$ e $\mathrm{CO}_{2}$. Os resultados de simulação apresentaram boa concordância com os dados experimentais.
\end{abstract}

\section{INTRODUÇÃO}

Segundo Gepts (2002), tamareira (Phoenix dactylifera) é uma palmeira de cultivo milenar e atualmente distribuída em regiões tropicais de todo o mundo. Registros históricos demonstram sua utilização por civilizações do Egito e Mesopotâmia desde 5000 a 6000 A.C. Seu fruto, a tâmara, é composta de $80 \%$ de açúcares, o restante consistindo de proteínas, gorduras e minerais, tais como cobre, enxofre, ferro e magnésio. Sua madeira também é aproveitada, assim como suas folhas, que são utilizadas na manufatura de cordas e cestos, o que demonstra a grande aplicabilidade desta planta.

Bassyouni et al. (2014) utilizaram folhas de tamareira como biomassa para a produção de gás de síntese a partir do processo de gaseificação. Tal processo envolve a oxidação parcial de uma fonte de carbono, como carvão, gás natural ou biomassa, originando produtos como $\mathrm{CO}_{2}, \mathrm{CH}_{4}, \mathrm{CO}$ e $\mathrm{H}_{2}$, sendo a mistura de $\mathrm{CO}$ e $\mathrm{H}_{2}$ também conhecida como gás de síntese.

O processo de gaseificação ocorre em gaseificadores, que podem ser classificados em: gaseificadores de leito fixo e de leito fluidizado. Dentre os gaseificadores de leito fixo, há diferentes configurações, de acordo com o sentido da corrente de ar em relação à corrente de alimentação de biomassa: updraft (fluxo contracorrente), downdraft (concorrente) ou crossflow (fluxo cruzado) (Ciferno e Marano, 2002). Os gaseificadores de leito fluidizado, por sua vez, são divididos entre gaseificadores de leito fluidizado borbulhante (LFB) e de leito fluidizado circulante (LFC).

As condições operacionais do processo de gaseificação, em geral, afetam a composição de saída da corrente de produtos. Por exemplo, Kumar et al. (2009) afirmam que o aumento da razão vapor-biomassa e o consequente aumento da pressão de vapor de água no sistema favorece as reações de formação de gás de síntese, de deslocamento gás-água e de reforma do metano, ocasionando um aumento da produção de $\mathrm{H}_{2}$. $\mathrm{O}$ aumento da temperatura, por sua vez, 
favorece as reações de formação de $\mathrm{H}_{2}$, que são endotérmicas. Na faixa de temperatura compreendida entre $850{ }^{\circ} \mathrm{C}$ e $900{ }^{\circ} \mathrm{C}$, as reações de Boudouard e de reforma a vapor são favorecidas com o aumento da temperatura, resultando no aumento da produção de CO.

\section{OBJETIVO}

O objetivo deste trabalho é simular o processo de gaseificação da biomassa de tamareira utilizando o software Aspen Plus, aprimorando um modelo já existente na literatura e verificando sua aplicabilidade. A influência da temperatura do gaseificador e da razão vaporbiomassa na composição dos produtos $\mathrm{H}_{2}, \mathrm{CO}, \mathrm{CO}_{2}$ e $\mathrm{CH}_{4}$, 'é avaliada.

\section{METODOLOGIA}

A simulação do processo de gaseificação de folhas de tamareira em um gaseificador de leito fixo do tipo downdraft foi realizada no Aspen Plus 8.4. O modelo utilizado neste trabalho foi uma modificação do modelo utilizado por Bassyouni et al. (2014), que realizaram experimentos de gaseificação da biomassa de tamareira paralelamente à execução de um modelo do processo construído com o auxílio do software Aspen Hysys.

A Figura 1 apresenta o fluxograma do modelo proposto neste artigo. A biomassa é alimentada a $8 \mathrm{~kg} / \mathrm{h}$ por meio da corrente BIOMASS, adentrando a zona de pirólise através do reator BM, um reator do tipo RYIELD no qual, por meio de um código Fortran, a biomassa é fracionada em seus principais constituintes: carbono, enxofre, hidrogênio, nitrogênio e oxigênio. Vapor é alimentado ao sistema por meio de duas correntes, STEAM1 e STEAM2, sendo a razão entre as vazões mássicas de STEAM2 e STEAM1 igual a 1,18. O separador COLUMN1 é responsável por separar componentes voláteis (corrente VOLATIL) do enxofre e sólidos (correntes SULFUR e SOLIDS, respectivamente) segundo a composição das folhas de tamareira apresentada por Bassyouni et al. (2014). A água não consumida no reator BM é eliminada do processo por meio da corrente de purga WATER. O reator VOLAT, um reator do tipo RGIBBS, é responsável pela combustão dos compostos voláteis. No reator GAS-A-1, modelo REQUIL, ocorrem as reações de oxidação completa do carbono $\left(\mathrm{C}+\mathrm{O}_{2} \rightarrow \mathrm{CO}_{2}\right)$ e de metanação $\left(\mathrm{C}+2 \mathrm{H}_{2}=\mathrm{CH}_{4}\right)$. No reator GAS-A-2, também de modelo REQUIL, ocorre a oxidação parcial do carbono $\left(\mathrm{C}+1 / 2 \mathrm{O}_{2} \rightarrow \mathrm{CO}\right)$. No reator GASB, do tipo REQUIL, ocorrem as reações de Boudouard $\left(\mathrm{C}+\mathrm{CO}_{2}=2 \mathrm{CO}\right)$, as de formação de amônia $\left(\mathrm{N}_{2}+3 \mathrm{H}_{2}=2 \mathrm{NH}_{3}\right)$ e de sulfeto de hidrogênio $\left(\mathrm{H}_{2}+\mathrm{S} \rightarrow \mathrm{H}_{2} \mathrm{~S}\right)$ e a formação do gás de síntese $\left(\mathrm{C}+\mathrm{H}_{2} \mathrm{O}=\mathrm{CO}+\right.$ $\left.\mathrm{H}_{2}\right)$. Em SHIFT, um reator REQUIL, ocorre a reação de deslocamento gás-água $\left(\mathrm{CO}+\mathrm{H}_{2} \mathrm{O}=\right.$ $\mathrm{CO}_{2}+\mathrm{H}_{2}$ ). O separador COLUMN2 é responsável por separar $\mathrm{H}_{2}, \mathrm{CO}, \mathrm{CO}_{2}$ e $\mathrm{CH}_{4}$ na corrente SYNGAS dos demais produtos.

Para a avaliação da influência da temperatura na composição de saída do gás de síntese, foi variada igualmente a temperatura dos reatores, de $650{ }^{\circ} \mathrm{C}$ a $800{ }^{\circ} \mathrm{C}$, mantendo-se constante a razão vapor-biomassa em 1,5. As vazões molares das correntes de saída de $\mathrm{H}_{2}, \mathrm{CO}, \mathrm{CO}_{2} \mathrm{e}$ $\mathrm{CH}_{4}$ foram registradas para cada temperatura.

Figura 1 - Fluxograma do modelo de gaseificação de biomassa de tamareira. 

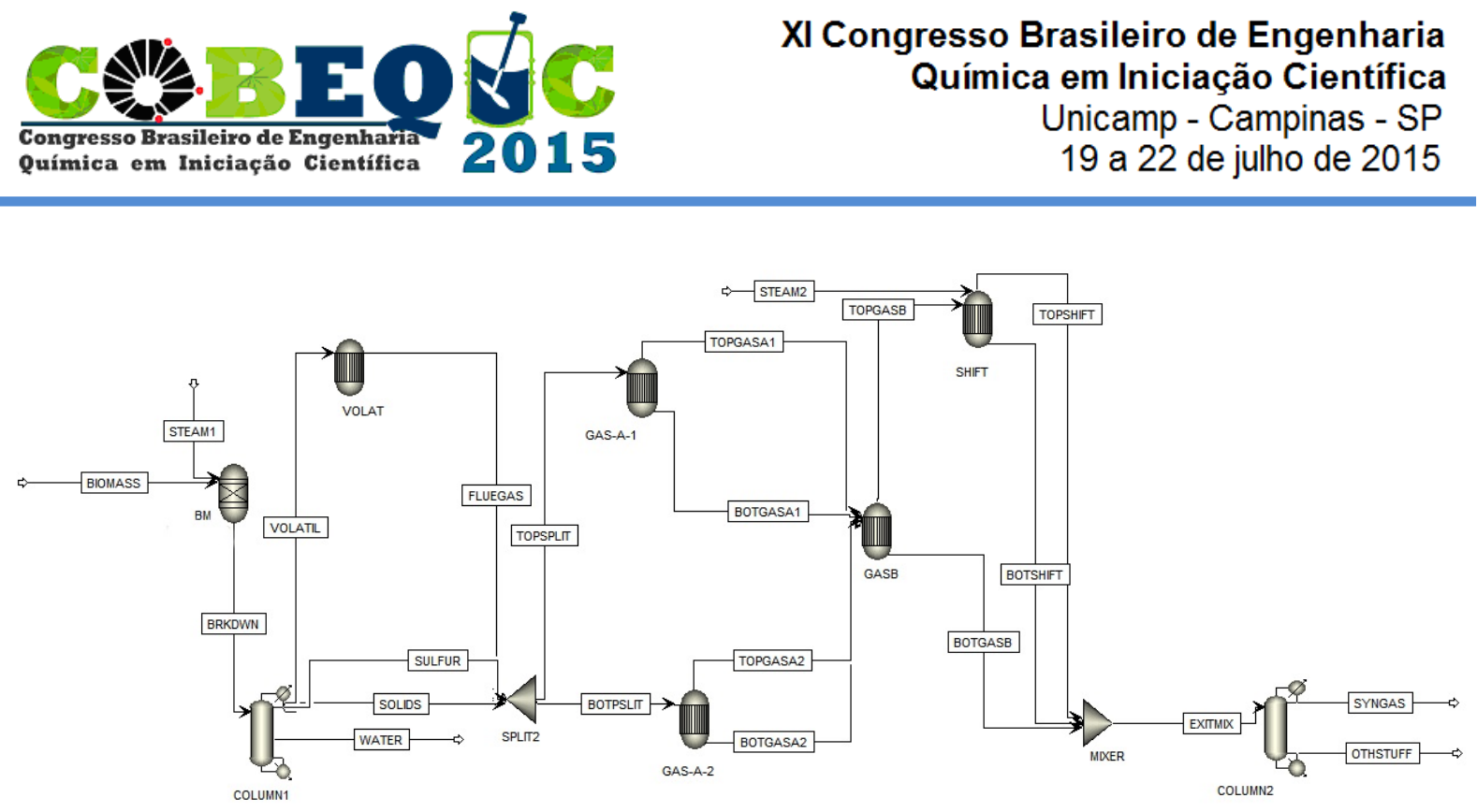

Analogamente à temperatura, foi variada a razão vapor-biomassa, de 0,5 a 2 , injetandose vapor através das correntes STEAM1 e STEAM2. Foi mantida igual a 1,18 a razão entre as vazões mássicas de STEAM2 e STEAM1. A temperatura dos reatores foi mantida a $800{ }^{\circ} \mathrm{C}$. Foram registradas as vazões molares das correntes de saída de $\mathrm{H}_{2}, \mathrm{CO}, \mathrm{CO}_{2}$ e $\mathrm{CH}_{4}$ para cada razão vapor-biomassa.

\section{APRESENTAÇÃO E DISCUSSÃO DOS RESULTADOS}

A Figura 2 apresenta os dados simulados do modelo proposto e os dados experimentais obtidos por Bassyouni et al. (2014) em relação à influência da temperatura do reator na composição dos produtos $\mathrm{H}_{2}, \mathrm{CO}, \mathrm{CO}_{2} \mathrm{e} \mathrm{CH}_{4}$. Pode-se observar que os dados obtidos do modelo ajustam-se, com grande precisão, aos dados experimentais, principalmente em relação ao $\mathrm{CO}$ e $\mathrm{CO}_{2}$. A tendência da curva de pontos simulados para o $\mathrm{H}_{2}$ não é a mesma dos pontos experimentais, diferentemente de $\mathrm{CO}$ e $\mathrm{CO}_{2}$; a curva de pontos simulados para o $\mathrm{CH}_{4}$ possui tendência mais similar à dos pontos experimentais conforme o aumento da temperatura.

O modelo proposto por Bassyouni et al. (2014) não obteve êxito ao simular o comportamento das curvas de $\mathrm{CO}$ e $\mathrm{CO}_{2}$ com o aumento da temperatura, cujos pontos destoaram-se dos pontos experimentais. Em relação aos gases hidrogênio e metano, o comportamento de ambas as curvas da simulação tornou-se mais similar ao das curvas experimentais conforme o aumento da temperatura. Portanto, o modelo modificado demonstrou ser mais preciso.

Pode-se observar na Figura 2 que o aumento da fração molar de $\mathrm{H}_{2}$ e $\mathrm{CO}$ na composição dos produtos ocorre com o aumento da temperatura. Este fenômeno deve-se ao fato de as reações de formação de gás de síntese e de Boudouard serem, segundo Bassyouni et al. (2014), endotérmicas, de entalpias de reação iguais a $+131 \mathrm{~kJ} / \mathrm{mol}$ e $+172 \mathrm{~kJ} / \mathrm{mol}$, respectivamente. Desse modo, ambas as reações, que possuem $\mathrm{CO}$ e $\mathrm{H}_{2}$ como produtos, são beneficiadas com o aumento da temperatura. Em relação ao $\mathrm{CO}_{2}$, sua formação ocorre em reações exotérmicas, que são desfavorecidas pelo aumento da temperatura, além de a substância estar presente como reagente na reação de Boudouard, que tende a consumir mais $\mathrm{CO}_{2}$ com o aumento da temperatura. O metano, por sua vez, é desfavorecido pelo aumento da 
temperatura, já que a reação de metanação é exotérmica $(\Delta \mathrm{H}=-74,8 \mathrm{~kJ} / \mathrm{mol})$, isto é, com o aumento da temperatura, o equilíbrio da reação é deslocado para a esquerda, consumindo $\mathrm{CH}_{4}$ e formando $\mathrm{C}$ e $\mathrm{H}_{2}$. Além disso, o deslocamento para a esquerda da reação de produção de amônia, exotérmica $(\Delta \mathrm{H}=-46,1 \mathrm{~kJ} / \mathrm{mol})$, com o aumento da temperatura também favorece a produção de $\mathrm{H}_{2}$.

Figura 2 - Variação da fração molar de $\mathrm{H}_{2}, \mathrm{CO}, \mathrm{CO}_{2}$ e $\mathrm{CH}_{4}$ com a mudança da temperatura do gaseificador à razão vapor-biomassa de 1,5.

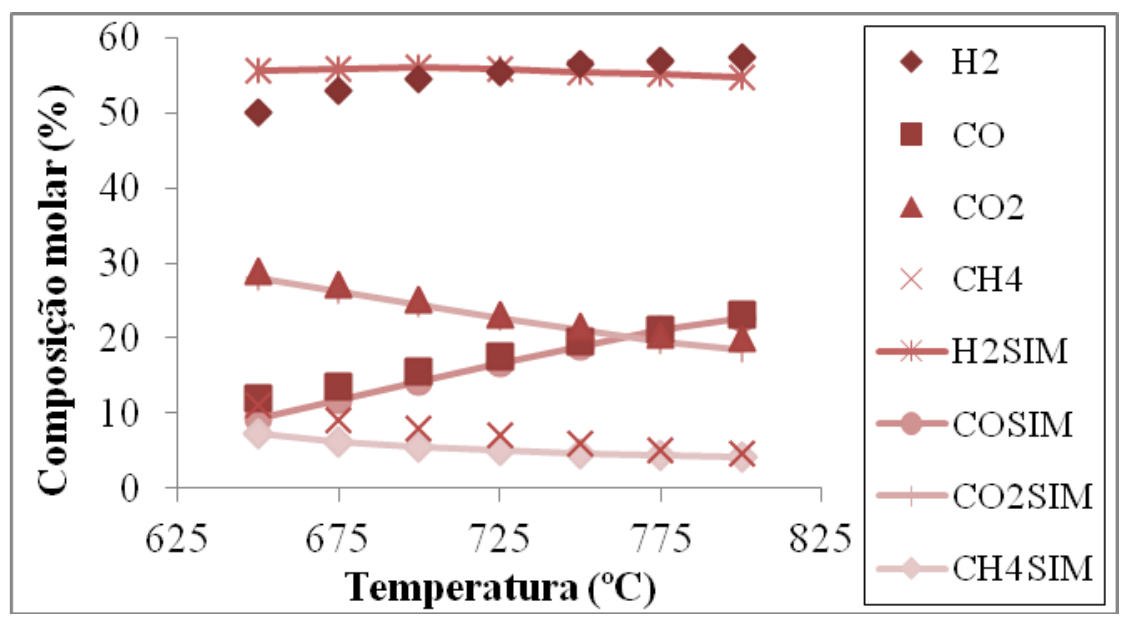

O efeito da variação da razão vapor-biomassa na composição dos produtos é apresentada na Figura 3. É possível verificar uma grande concordância entre os dados da simulação e os dados experimentais, principalmente em relação a $\mathrm{H}_{2}, \mathrm{CO}$ e $\mathrm{CO}_{2}$, na faixa de razões vapor-biomassa analisadas. As curvas relacionadas ao $\mathrm{CH}_{4}$, exibidas na Figura $3 \mathrm{~d}$, apresentam comportamento mais similar conforme aumento da razão vapor-biomassa.

O modelo de Bassyouni et al. (2014) obteve resultados satisfatórios em relação à composição de saída dos produtos como função da razão vapor-biomassa. Os dados ajustaram-se com maior concordância para $\mathrm{H}_{2}$ e $\mathrm{CO}$. Quanto ao $\mathrm{CO}_{2}$, a concordância entre pontos da simulação e pontos experimentais foi maior conforme aumento da razão vaporbiomassa. Para o metano, no entanto, o modelo não obteve boa precisão.

O aumento da razão vapor-biomassa foi feito com o aumento da vazão de vapor a uma vazão constante de biomassa na alimentação. A água é consumida nas reações de formação de gás de síntese e no deslocamento gás-água. $\mathrm{O}$ aumento da concentração desse reagente no processo provoca aumento da formação de produtos. No caso da reação de formação de gás de síntese, $\mathrm{CO}$ e $\mathrm{H}_{2} \mathrm{O}$ são formados, porém, $\mathrm{CO}$ também é reagente na reação de deslocamento gás-água, que promove a formação de $\mathrm{CO}_{2}$ e $\mathrm{H}_{2}$. Isto poderia explicar o crescimento significativo apenas da fração molar de $\mathrm{CO}_{2}$ com o aumento da razão vaporbiomassa. $\mathrm{CO}$, sendo consumido na reação de deslocamento gás-água, tem sua fração molar diminuída conforme o aumento da razão vapor-biomassa. O gás hidrogênio, por sua vez, não possui uma taxa de aumento tão significativa quanto o $\mathrm{CO}_{2}$, visto que, apesar de ser produzido na reação de deslocamento gás-água e na formação de gás de síntese, é também um 


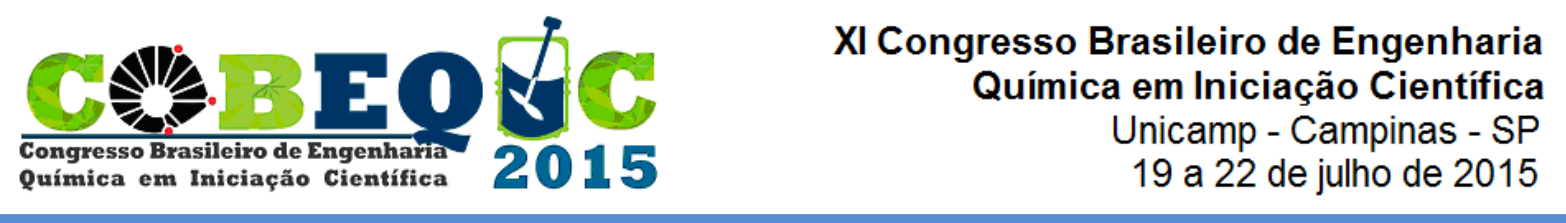

dos reagentes na produção de metano por meio da reação de metanação. Dessa forma, a produção do metano também é favorecida, como exibido na Figura 3d.

Figura 3 - Variação da fração molar dos produtos da gaseificação com a razão vaporbiomassa à temperatura do reator de $800^{\circ} \mathrm{C}$ : a) gás hidrogênio; b) dióxido de carbono; c) monóxido de carbono; d) metano.
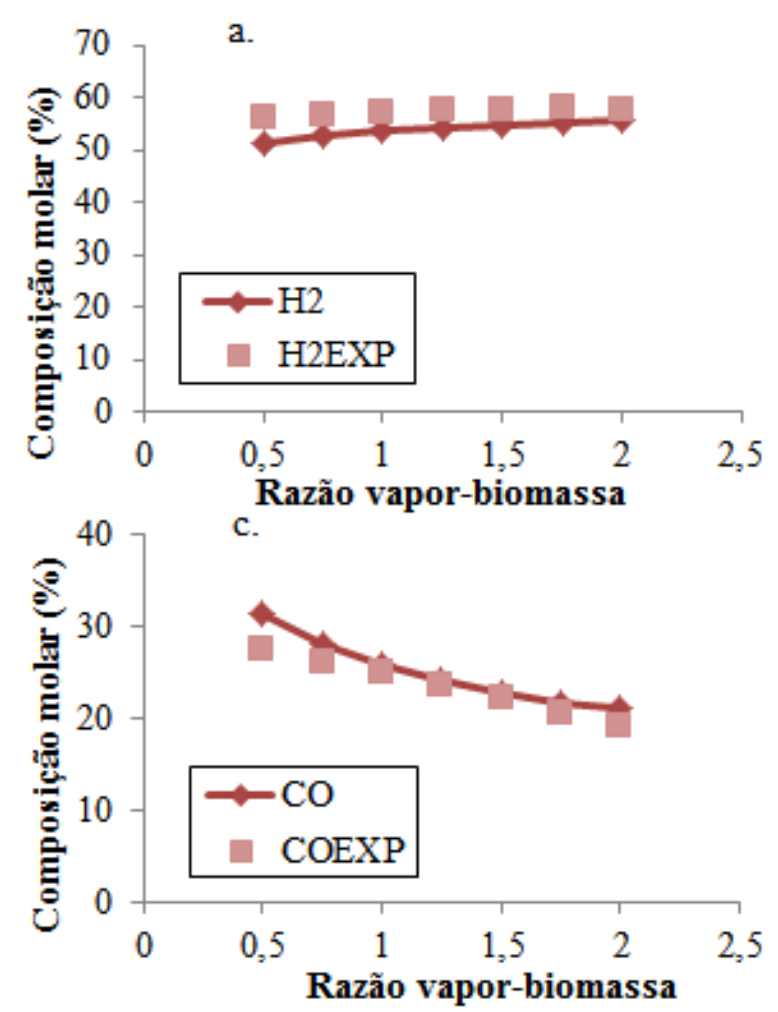

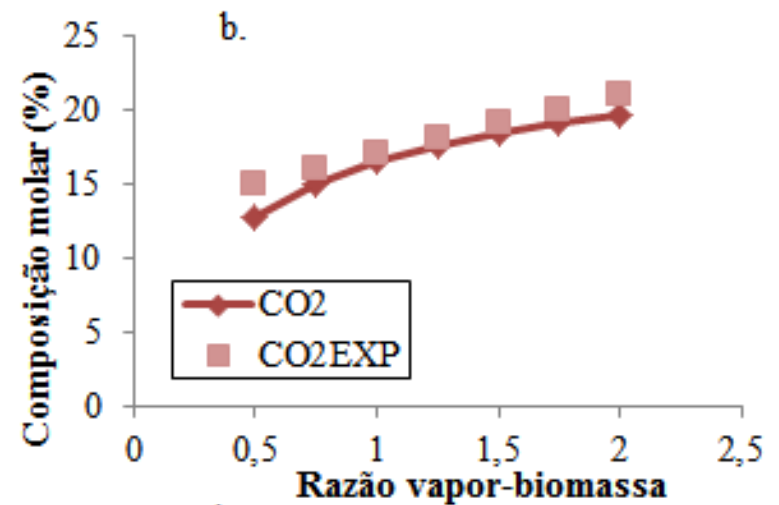

d.

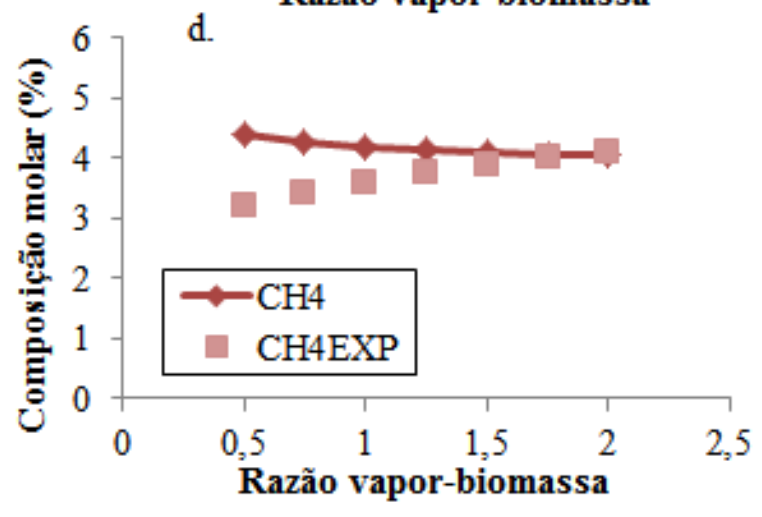

\section{CONCLUSÃO}

O modelo modificado obteve bastante eficácia na simulação do processo de gaseificação de biomassa de tamareira em gaseificador downdraft, na faixa de temperaturas e razões vapor-biomassa analisadas, representando melhor o processo que o modelo original.

$\mathrm{O}$ aumento da temperatura favoreceu os produtos das reações endotérmicas $\left(\mathrm{CO}\right.$ e $\left.\mathrm{H}_{2}\right)$, ao mesmo tempo que ocasionou diminuição da fração molar dos produtos de reações exotérmicas e reagentes das reações endotérmicas $\left(\mathrm{CO}\right.$ e $\left.\mathrm{CH}_{4}\right)$.

$\mathrm{O}$ aumento da razão vapor-biomassa ocasionou aumento da produção de $\mathrm{CO}$ e $\mathrm{H}_{2}$ através da reação de formação de gás de síntese e aumento da produção de $\mathrm{CO}_{2}$ por meio da reação de deslocamento gás-água. Entretanto, por participar da reação de deslocamento gáságua como reagente, o monóxido de carbono sofreu um efeito global de redução de sua fração molar na mistura de saída conforme o aumento da razão vapor-biomassa. A reação de metanação também foi favorecida pelo aumento da razão vapor-biomassa, gerando aumento da fração molar de metano e contendo o aumento da fração molar de gás hidrogênio no gás de síntese. 


\section{REFERÊNCIAS}

BASSYOUNI, M.; HASAN, S. W. ul; ABDEL-AZIZ, M. H.; HUSSAIN, A.; ANI, F. N. Date palm waste gasification in downdraft gasifier and simulation using ASPEN HYSYS. Energy Conv. and Manag., v. 88, p. 693 - 699, 2014.

CIFERNO, J. P.; MARANO, J. J. Benchmarking biomass gasification technologies for fuels, chemicals and hydrogen production. In: U.S. Department of Energy/National Energy Technology Laboratory, 2002.

GEPTS, P. The crop of the day: The Date, Phoenix dactylifera. [s.1., 2002]. Disponível em: http://www.plantsciences.ucdavis.edu/GEPTS/pb143/CROP/DATE/date.htm. Acesso em: 18 fev. 2015.

KUMAR, A.; JONES, D. D.; HANNA, M. A. Thermochemical biomass gasification: a review of the current status of the technology. Energies, vol. 2; p. 556 - 581, 2009.

SCHEFFLAN, R. Teach yourself the basics of Aspen Plus. Hoboken: John Wiley \& Sons, 2011. 